\title{
A Complex Network Model of Student Cultivation Quality Evaluation Base on Critique Each Other
}

\author{
Qingyu Zou ${ }^{1, a}$, Dejun Liu ${ }^{1, b^{*}}$ and Huida Duan ${ }^{1, c}$, Liyun Xing ${ }^{1, d}$ \\ ${ }^{1}$ College of Electrical and Information Engineering of Beihua University, Jilin, China \\ azouqingyu2002@126.com, bdejunliu@126.com, chuida_duan@126.com, \\ xingliyun116@foxmail.com \\ *The corresponding author
}

Keywords: Complex network; Network model; Students cultivation quality evaluation; Critique Each Other

\begin{abstract}
The student cultivation quality evaluation is playing a more and more important role, as an important means of teaching quality supervision. In essence, the problem can be regarded as a multiple attribute decision making problem, which includes both qualitative and quantitative attributes. The characteristics of complex network model determine that it can be used to solve this problem. In this paper, the complex network model of college student cultivation quality evaluation had been established by the evaluation system of students, and then the structure characteristics of the network model are used to measure the quality of student cultivation. We use this model to evaluate the cultivation quality of 39 electronic information engineering students. The results show that this complex network model can accurately measure the cultivation quality of the students of electronic information engineering.
\end{abstract}

\section{Introduction}

Higher education is shouldering the important mission of cultivating high-quality and top-notch innovative talents. With the continuous reform of the teaching system in colleges and universities, the strategic core of the development of colleges and universities is more people-oriented, and pay more attention to a full range of student cultivation. The evaluation of the quality of student cultivation can help the managers to find out the gap between the actual situation and the educational goal. Research on the quality of student cultivation had sprung up from 1980s on. The main research are focused on the quality standards of university student cultivation, the quality evaluation of university student cultivation, the establishment of the quality assurance system of university student cultivation and so on. At present, there are many methods to evaluate the quality of student cultivation at home and abroad. Including PB neural network, HPA, gray correlation analysis, etc. The neural network to imitate the structure of the human brain mapping input features and the output of the conclusion of the nonlinear relationship, but its input and output are required to be accurate numerical. HPA is a complex problem with multiple objectives, multiple criteria, multiple factors and multiple levels. It is a method that can carry on qualitative and quantitative system analysis, decision analysis and comprehensive analysis. Grey incidence analysis method has the advantages of clear evaluation criteria and relatively objective results, but it is a quantitative evaluation method, which can not completely describe the evaluation object. Complex network theory can be used to fuse uncertain qualitative attributes and quantitative attributes, so that the results are more in line with the actual situation. In this paper, the complex network theory is applied to the quality evaluation of student cultivation in colleges and universities, and puts forward a method based on complex network theory to evaluate student cultivation quality [1-5]. 


\section{Evaluation Criteria System for Student Cultivation Quality}

The design and establishment of index system is the basic work of college student cultivation quality evaluation. In view of the actual situation of the quality management of talents training in Colleges and universities, we have carried out research on some universities, and constructed a comprehensive evaluation index system of college student cultivation quality, which including five aspects of physical condition: understanding the degree of innovation and practical ability, moral level, cooperation and coordination capacity. Degree of understanding is the degree of familiar of students evaluated by evaluating students. Moral quality is the comprehensive evaluation of ideological and moral and professional ethics of students evaluated by evaluating students. The ability of innovation practice is the comprehensive evaluation of theory, practice, application knowledge, independent thinking, innovation consciousness and innovation ability of students evaluated by evaluating students. Basic theory level is the comprehensive evaluation of public knowledge, professional basic knowledge and professional knowledge of students evaluated by evaluating students. The ability of cooperation and coordination is the evaluation of the ability to communicate and cooperate with colleagues or partners, and to correctly handle the internal and external relations of the organization. Physical condition refers to the health of students, physical condition, and the comprehensive evaluation of sports skills of students evaluated by evaluating students.

\section{Dynamic Evolution of Network Model}

Distance threshold plays an important role in the modeling process. The complex network model is built based on different distance thresholds, the network topology and the network characteristics are not the same, and this has an important impact on the proposed identification method. The corresponding network model can provide more details for smaller distance threshold. On the contrary, the corresponding network model can provide more global information, but it has lost some useful information. In order to summarize the overall information and details and fully reflect the characteristics of the recognition object, we need a dynamic network modeling and parameter extraction method. Therefore, the actual modeling process is not using a single distance threshold static modeling method, but multiple distance thresholds. It is a dynamic multiple complex network model. Network topology change with the distance threshold and the changes in the process can also be viewed as a function of time, at different times, different distance threshold value, corresponding to different network model, so modeling process can also as a dynamic evolution process [6-9].

\section{Structure Characteristic of Network: Hub and Authorities}

In the case of directed networks, it is appropriate to accord a vertex high centrality if it points to others with high centrality. There are really two types of important node in these networks: authorities are nodes that contain useful information on a topic of interest; hubs are nodes that tell us where the best authorities are to be found. An authority may also be a hub, and vice versa: review articles often contain useful discussions of the topic at hand as well as citations to other discussions. Clearly hubs and authorities only exist in directed networks, since in the undirected case there is no distinction between pointing to a vertex and being pointed to [10].

The authority centrality of a vertex is defined to be proportional to the sum of the hub centralities of the vertices that point to it:

$$
x_{i}=\alpha \sum_{j} A_{i j} y_{j}
$$

Where $\alpha$ is a constant. Similarly the hub centrality of a vertex is proportional to the sum of the authority centralities of the vertices it points to: 


$$
y_{i}=\beta \sum_{j} A_{j i} x_{j}
$$

Where $\beta$ another constant. Notice that the indices on the matrix element $A_{j i}$ are swapped around in this second equation: it is the vertices that $i$ points to that define its hub centrality.

\section{Result of Application}

We use this method for the student cultivation quality evaluation of a class. The numbers of students in class are 39. We build an initial network model of student cultivation quality evaluation using this method. The initial network model consists of 39 nodes and 780 links. Fig. 1 shows the resulting initial network model of student cultivation quality evaluation. In this network, nodes represent the students in class and links represent the evaluation weights between them. The links are directional, which direction from appraiser to evaluation objectives. The dynamic evolution network model derived from the initial network model as shown in Fig. 2 consists of 39 nodes and 307 links.

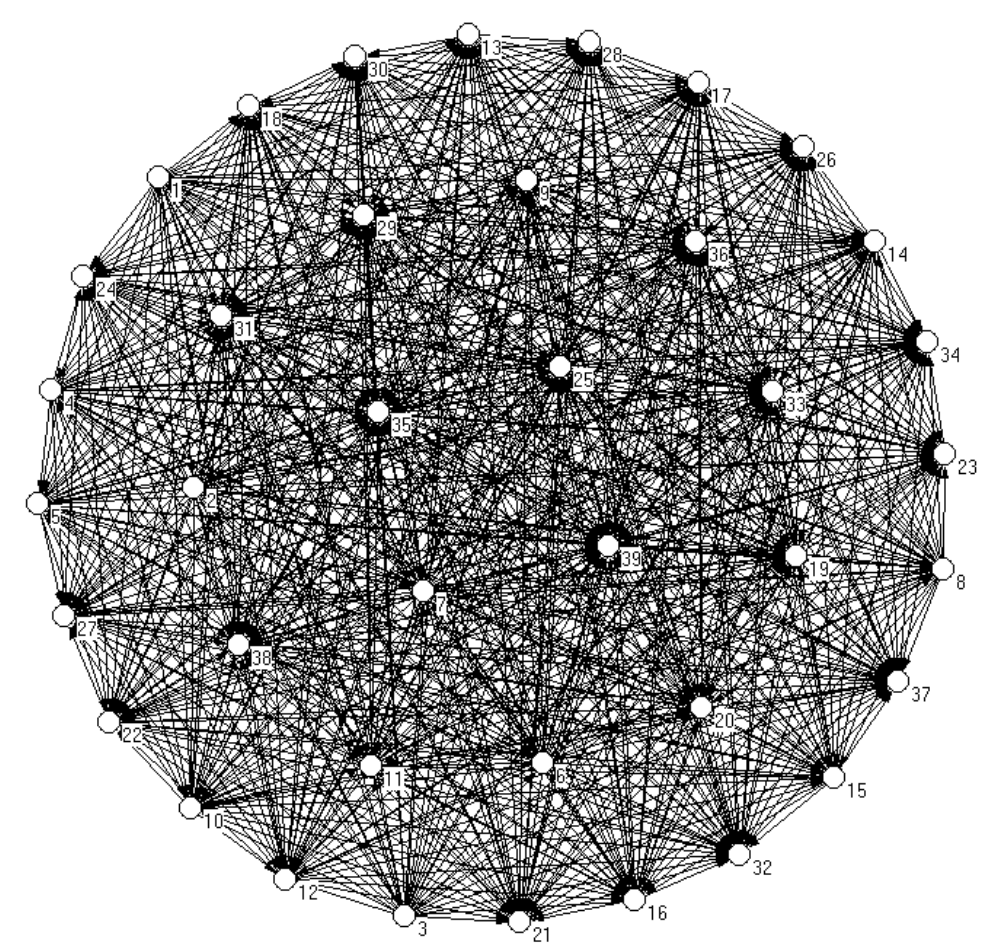

Figure 1. Initial network model of student cultivation quality evaluation

Hub. Although hub centrality is a simple centrality measure, it can be very illuminating. In the student cultivation quality evaluation network, hub of a node could give a measure of whether the student has been approved or not and is used as a metric for judging the learning effectiveness. The distributions and cumulative distributions of hub of student cultivation quality evaluation network of this class as in Fig. 3. As can be seen from the figure, the cumulative distribution of hub are large radian and the distribution of hub follows a normal distribution. This means that the vast majority of students received similar learning outcomes, while there is a small received excellent learning outcomes.

Authorities. Authorities measures the learning situation of vertices. Fig. 4 shows the distributions and cumulative distributions of authorities of student cultivation quality evaluation network. As can be seen from the figure, the distribution of authorities follows a normal distribution. We find that in the largest component of the network, which includes more than $90 \%$ of all students. 


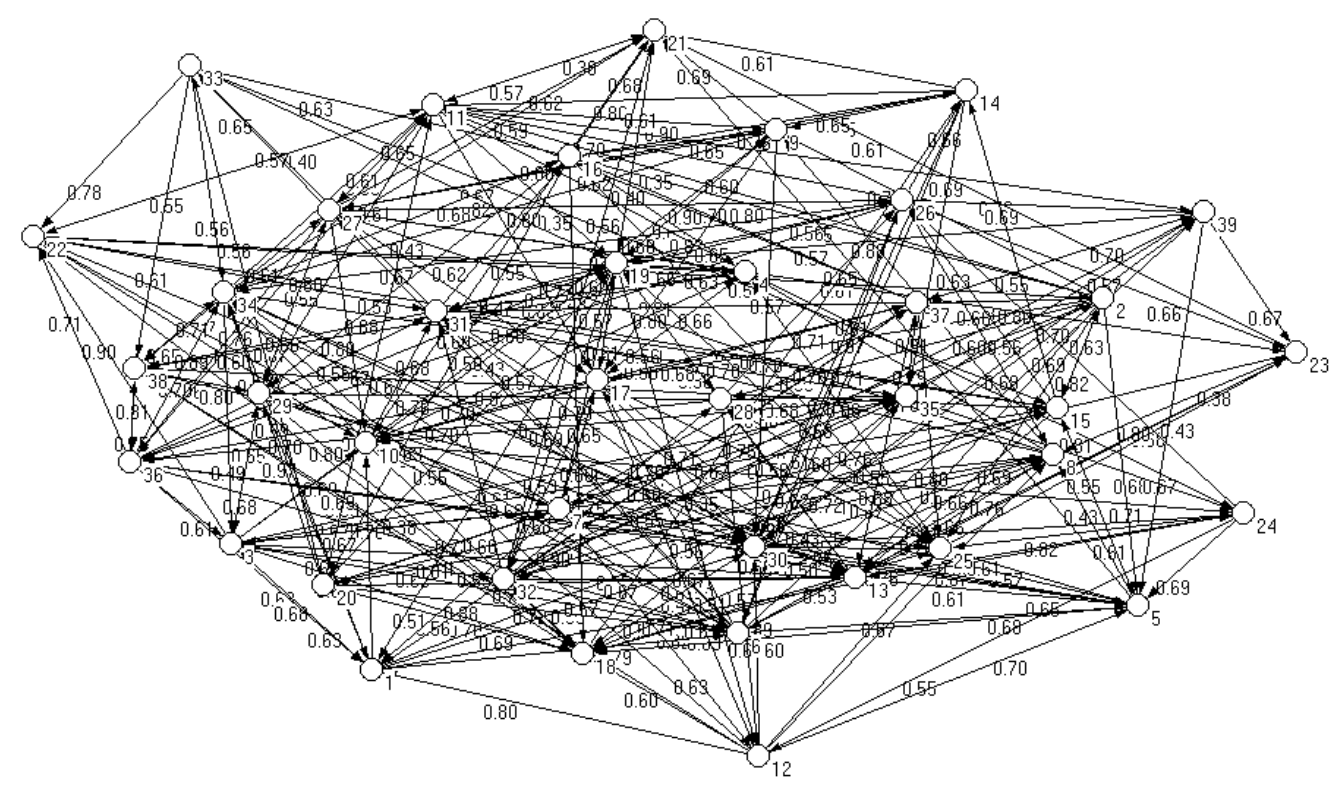

Figure 2. Dynamic evolution network model of student cultivation quality evaluation
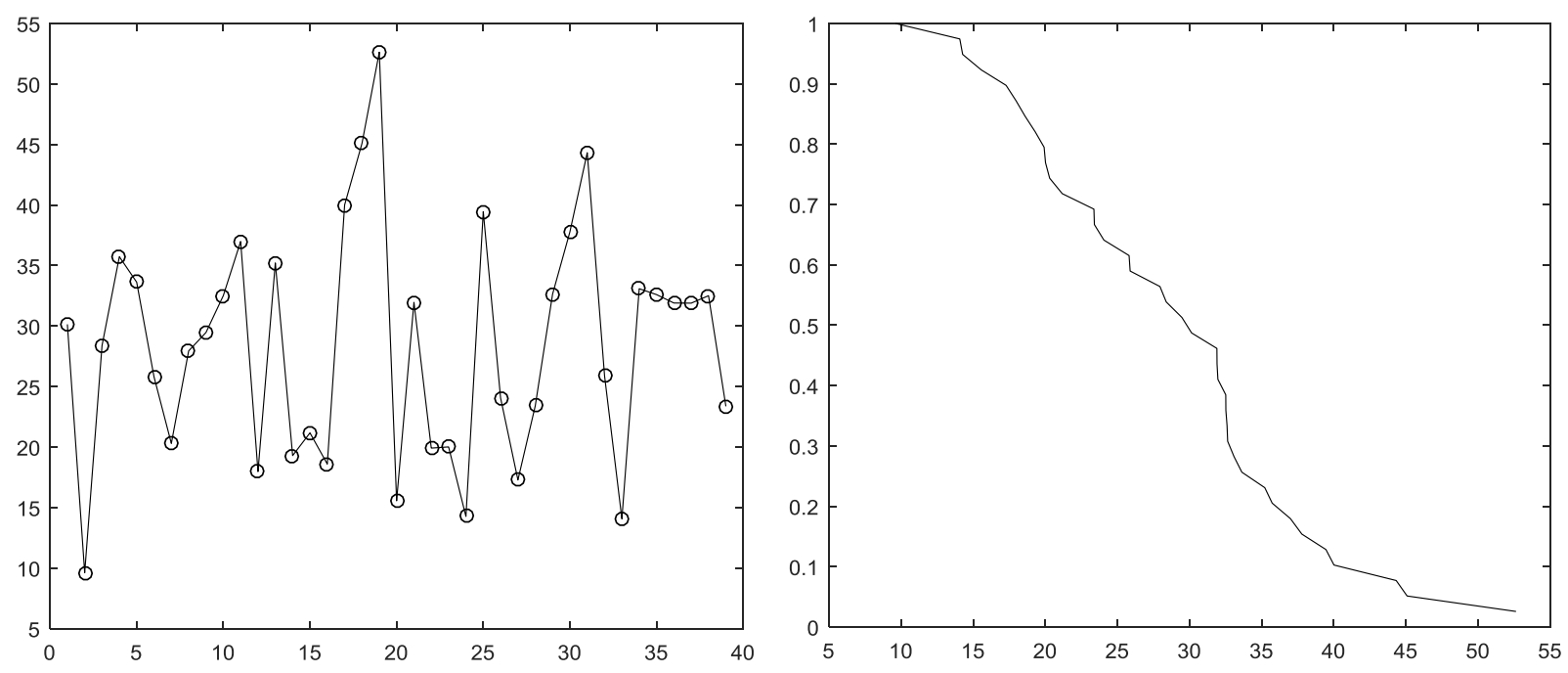

Figure 3. Distributions and cumulative distributions of hub of dynamic evolution network model of student cultivation quality evaluation.

\section{Summary}

The student cultivation quality evaluation network will be useful to evaluate the quality of colleges and universities students training. Building a student cultivation quality evaluation network model is a guarantee to promote the students cultivation quality management in higher vocational colleges. Establishing a high quality of student cultivation evaluation network model is directly related to the healthy development of the vocational education. It is the inevitable developing trend of scientific management in the 21 st century education and is inevitable requirement of education facing the modernization, the world and the future. 

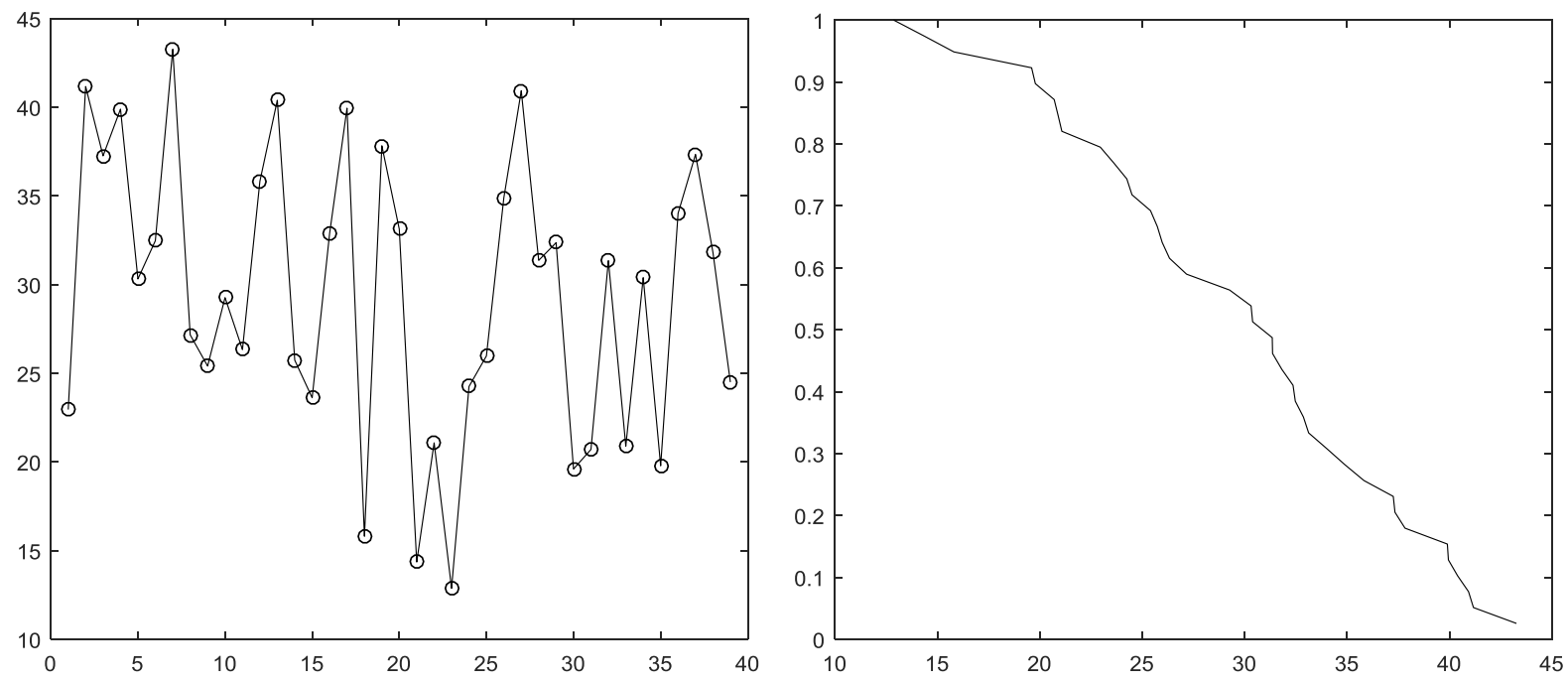

Figure 4. Distributions and cumulative distributions of authorities of dynamic evolution network model of student cultivation quality evaluation.

\section{Acknowledgements}

This work is supported mostly by the Key Education Research Project of Beihua university, partially by the Jilin Province department of education Science and Technology Plan Projects. (Grant NO 2015161, Grant NO. 201658), partially by the Beihua University Dr. Scientific Research Fund (Grant NO. 199500103) and partially by the national professional comprehensive reform pilot project (Grant NO. ZG0119).

\section{References}

[1] Z. Wei: China Higher Education Research, Vol.12 (2012) No.09, p.67.

[2] H. Zixiang: Modern University Education, Vol.2006 (2006) No.02, p.61.

[3] M.A. O' NeillandA: Palmer Quality As surance in Education, Vol.12 (2004) No.1, p.37.

[4] S. Lagrosen: Quality As surance in Education, Vol.12 (2004) No.2, p.61.

[5] P. Zhou: Proceedings of the 10th Euro-Asia Conference on Environment and Corporate Social Responsibility: Tourism, Society and Education Session, Pt. Ii, Vol. (2014) p.158.

[6] P.M. Sato, K. Yoganathan, J.H. JungandS. G. Peisajovich: Plos Biology, Vol.12 (2014) No.12.

[7] G.A. PaganiandM. Aiello: Physica a-Statistical Mechanics and Its Applications, Vol.396 (2014) p. 248 .

[8] P. Braun, A. - R. Carvunis, B. Charloteaux, et al.: Science, Vol.333 (2011) No.6042, p.601.

[9] N.D. Martinez: Network evolution: Exploring the change and adaptation of complex ecological systems over deep time, 2006).

[10] M.E.J.Newmen: Networks: An Introduction (Oxford University Press, New York 2010) 